\title{
Personalized Diabetes Clinics Revisited
}

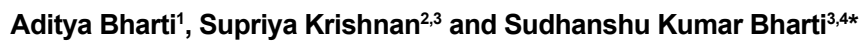

${ }^{1}$ Department of Computer Science and Engineering, NMAM Institute of Technology, Nitte-574110, Karnataka, India ${ }^{2}$ Department of Psychology (PMIR), Patna University, Patna-800005, Bihar, India

${ }^{3}$ Department of Biochemistry, Patna University, Patna-800005, Bihar, India

${ }^{4}$ Synergy Diabetes Research Foundation, Patna-800023, Bihar, India

\section{Editorial}

Precision medicine (PM) is a contemporary therapeutic model en route for elucidating customized perfect medical treatments approach to an individual patient according to the molecular and genomic profiling and systems of biological components involved in the patient's disease process. PM is a grown-up and preferred term of 'personalized medicine' with a similar meaning as the word 'personalized' which may possibly be misconstrued to entail that disease interventions are exclusively for each patient while PM intervention strategies accentuate to make out appropriate and effective approaches based on patient's genetic, environmental and lifestyle factors [1].

The deep-seated goal of medical sciences is to mine the information at molecular level to create new opportunities to detect and monitor the molecular databases of disease and disease vectors embedded in genomes, epigenomes and proteomes to facilitate the phenotypic (diseases) correlation of the clinical implications. This include genome-wide association study (GWAS), phenome-wide association study (PheWAS), epigenetics and large data sets of protein-protein interactions (PPI) which can be modelled as PPI networks, technologies generating big medical data, big data storage and maintenance, data integration, analysis and visualization with big data sharing tools and policies, management, interpretation and application of big data in diseases and explore the challenges and roadblocks for moving beyond into health [2]. With more and more patients submitting their genome for clinical studies the size of 'omics dataset' and 'clinical dataset' are exploding which eventually empower the precision medicine concept. This data-flood provides an opportunity to extract a complete picture of gene-disease association for multifactorial diseases like diabetes.

Diabetes mellitus is a heterogeneous group of disorders characterized by persistent hyperglycemia [3]. The genetic, environmental and lifestyle factors involved with the development of diabetes have been identified. For instance, the genetic basis of most common forms of diabetes i.e., type 1 diabetes [4] and type 2 diabetes [5] have been well documented without complete understanding of molecular basis of pathophysiology. In addition, sedentary lifestyle, unctuous diet and other environmental factors have also been recognized to be involved in pancreatic $\beta$ cell disfunction, reduced insulin action/ glucose metabolism, or other metabolic conditions (e.g., energy intake/ expenditure, lipid metabolism) [3]. Despite optimal medical therapy, only a few numbers of patients demonstrate a poor control of the disease and experience repeated and unpredictable lapses of blood glucose levels to both ends of the spectrum ranging from severe hypoglycaemia to uncontrolled hyperglycaemia, resulting in severe and potentially grave situation.

Currently, we are experiencing ever-increasing number of patients of all ages suffering from diabetes. The increasing medical burden of patients with diabetes related complications also results in an enormous economic burden, which could severely impair global economic growth in the near future [3]. This shows that today's health system has conventionally been poorly equipped towards confronting the mounting impact of diabetes on a global scale and beseeches an urgent need for newer and better options in the form of 'precision diabetes clinics. The overall challenge to the field of precision medicine as it relates to diabetic treatment is to identify the individualized factors that can lead to improved glycemic control.

A model precision diabetes clinic will gather omics, clinical, genomic epidemiological and behavioural data of an individual diabetic patient; effectively integrate and efficiently analyze them over a period of time and can answer many of the impending diabetic problems as well as provide lifestyle counselling and genomic-based pharmacotherapy where appropriate based on an extensive set of personalized data analysis [6]. By the use of diabetes technology gadgets and mHealth tools, the reengineered futuristic multidisciplinary precision diabetes clinics would potentially provide novel therapeutic options that are more coordinated, proactive and patient-centric through specialized and customised cost-effective prevention/ treatment approach. The clinic would be equipped to construe wirelessly transmitted data of glycemic patterns of patients outside of clinical setting delivered by remote-monitoring technologies (videoconferencing, telephone-, internet- and mobile-based systems) whilst patients would be given access to technology-based interventions of self-management tools with data gathering mechanisms for maintaining their normoglycemic level either through web- or mobile- based tools (Figure 1). However

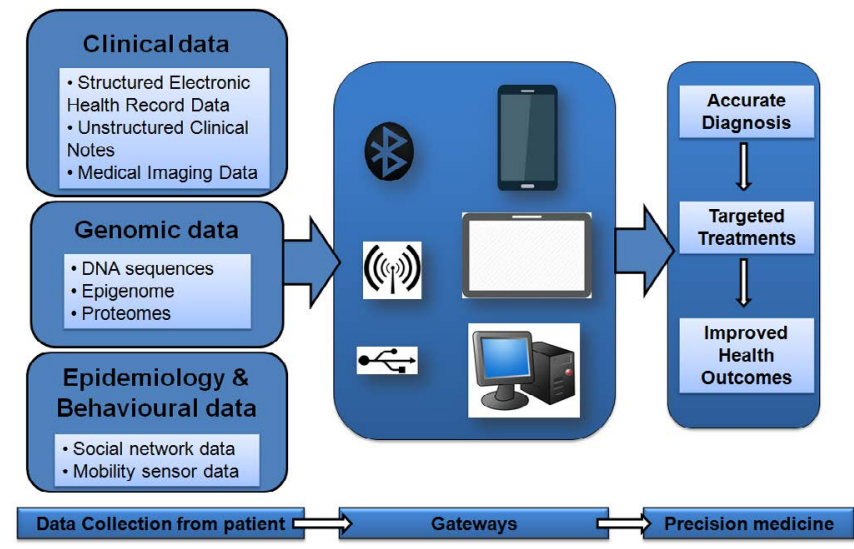

Figure 1: Sources and practices implicated in precision diabetes clinics.

*Corresponding author: Sudhanshu Kumar Bharti, Department of Biochemistry, Patna University, Patna-800005, Bihar, India, Tel: +91-9852883752; E-mail: sudhanshu_bharti@rediffmail.com

Received: August 25, 2015; Accepted: August 26, 2015; Published August 28 , 2015

Citation: Bharti A, Krishnan S, Bharti SK (2015) Personalized Diabetes Clinics Revisited. Biochem Anal Biochem 4: e159. doi:10.4172/2161-1009.1000e159

Copyright: ( 2015 Bharti A, et al. This is an open-access article distributed under the terms of the Creative Commons Attribution License, which permits unrestricted use, distribution, and reproduction in any medium, provided the original author and source are credited. 


\section{S.}

No. Barriers to the success of precision diabetes clinics

1. Technical barriers

The clinic to be equipped with advanced and updated

a. Investigation diagnostic tools to investigate and construe diverse multiple big databases to reveal significant insights.

\begin{tabular}{l|l|l} 
b. Usability & The clinic using big data to have a good user gateway \\
interface for data mining to efficiently decode enquiry
\end{tabular} and then swiftly and accurately code outcomes.

\begin{tabular}{l|l|l}
\hline c. & The confidentiality of the sources of data concerning \\
clinic patients composing big database must be
\end{tabular} assured.

d. Safety $\quad$ The safety of the sources of data concerning clinic

2. Clinical barriers patients and their outcomes must be assured.

\begin{tabular}{|l|l|l|}
\hline a. & Treatment-precision & $\begin{array}{l}\text { The phenotypic and behavioural data provided } \\
\text { by sensors and other digital gadgets can lead to } \\
\text { controlled treatment plans but lack consensus on } \\
\text { treatment protocols on patients with many genetic } \\
\text { and genomic variants. The reliability and accuracy of } \\
\text { sensors and gadgets are also a concern. }\end{array}$ \\
\hline b. & Expenses & $\begin{array}{l}\text { The cost of genomic analysis and interpretation are } \\
\text { still very high. }\end{array}$ \\
\hline c. & Reimbursement & $\begin{array}{l}\text { There is a lack of transparent policy for reimbursement } \\
\text { for this new approach. }\end{array}$ \\
\hline d. & Accuracy & $\begin{array}{l}\text { In type } 2 \text { diabetes, the candidate genes exhibit a } \\
\text { petite increased risk compared to unhealthy behaviour } \\
\text { as well as variants of ethnicity and hence dynamicity } \\
\text { of biomarkers should be made accurate than static } \\
\text { measurements for calculating individual's risk. }\end{array}$ \\
\hline
\end{tabular}

Table 1: Barriers to the success of precision diabetes clinics.

there are several potential clinical and technical barriers will have to be overcome for precision diabetes clinics to be successful (Table 1) [7]. The self-management websites facilitate patient's daily diabetes care where the patient can access their information, input their data and track their progress. Self-management mostly involves the adjustments of diet and exercise and the use of medication or insulin to control blood glucose. Internet and mobile applications offer variety of tools such as electronic diaries, medication reminders, calculators for insulin dose adjustment or carbohydrate counting, food databases for nutrients optimisation at meals, and tracking of physical conditions.

\section{Conclusions}

Precision medicine clinics pledges to provide a platform for the integration of different biological techniques, statistical analysis methods and information technologies to translate basic molecular, genetic, cellular and clinical data into clinical applications to classify diseases into subsets, all with their own best treatments to improve each patient's health. Precision medicine clinics will channelize efficient analysis of big medical database for a better understanding of diseases especially complex diseases like diabetes at molecular level and will use the emerging data streams to deliver individualized treatments for patients with the development of predictive, preventive and better precision diagnostics and therapeutics. While diabetes afflicts millions of people and can place a tremendous clinical and financial burden on our society, precision diabetes clinics offers an opportunity to improve care processes that enhance the lives of patients and help control the medical costs associated with it.

\section{References}

1. Katsnelson Alla (2013) Momentum grows to make 'personalized' medicine more 'precise'. Nature Medicine 19: 249.

2. Merelli I, Pérez-Sánchez H, Gesing S, D’Agostino D (2014) Managing analysing and integrating big data in medical bioinformatics: open problems and future perspectives. Biomed Res Int: 134023.

3. Bharti SK, Krishnan S, Gupta AK (2013) Herbal formulation to combat type 2 diabetes mellitus, LAMBERT Academic Publishing, Germany.

4. Santin I, Eizirik DL (2013) Candidate genes for type 1 diabetes modulate pancreatic islet inflammation and $\beta$-cell apoptosis. Diabetes Obes Metab 15:71-81.

5. Abbas S, Raza ST, Ahmed F, Ahmad A, Rizvi S, et al. (2013) Association of genetic polymorphism of PPARY-2, ACE, MTHFR, FABP-2 and FTO genes in risk prediction of type 2 diabetes mellitus. J Biomed Sci 20: 80.

6. National Research Council Committee on a Framework for Developing New Taxonomy of Disease (2011) Toward Precision Medicine: Building a Knowledge Network for Biomedical Research and a New Taxonomy of Disease. Washington, DC: National Academies Press.

7. Klonoff DC (2015) Precision Medicine for Managing Diabetes. J Diab Sci Technol 9: 3-7. 